\title{
A NOVEL MUTATION AFFECTING THE COPA GENE:THE FIRST REPORT OF COPA SYNDROME IN BRAZIL
}

\author{
Teresa Cristina Martins Vicente Robazzi ${ }^{1, *}$, Regina Ramos Terse ${ }^{1}$, Leonardo Oliveira Mendonça ${ }^{2}$ \\ 1. Universidade Federal da Bahia, Salvador (BA), Brazil. 2. Universidade de São Paulo, São Paulo (SP), Brazil. \\ *Corresponding author: trobazzi.ufba@gmail.com
}

\section{BACKGROUND}

Protein transport between the endoplasmic reticulum and Golgi compartments is mediated in part by nonclathrin-coated vesicular coat proteins (COPs). The COPA gene disrupts the alpha subunit of this complex. Immunologically, this disequilibrium culminates in unfolded proteins in the cytoplasm and, consequently, cellular stress with marked type-1 interferon secretion. In patients who harbor pathogenic COPA mutations, some manifestations, such as severe destructive arthritis, occur early in life, while interstitial lung involvement develops later. Patients present positive rheumatoid factor and ANCA antibodies. Here, we describe the first case of COPA syndrome reported in Brazil, characterized by a novel mutation in the COPA gene.

\section{RESULTS}

A 16-year-old adopted boy from the Northeastern Region of Brazil came to our attention due to chronic arthritis in association with dyspnea. The onset of arthritis symmetrically affecting multiple large joints reportedly occurred at six years of age. High levels of acute phase reactants, rheumatoid factor and ANCA antibodies were detected. High doses of steroids and methotrexate were somewhat effective in controlling joint inflammation, but the relapse of arthritis and subclinical progressive joint damage were observed during the course of disease. At age 12 , his dyspnea worsened and lung CT presented ground-grass aspect predominantly in the lower lobes, in addition to multiple cystic formations. Due to the suspicion of inborn error of immunity, commercially available exome sequencing (Fleury genomics) was requested. Single exome sequencing revealed a novel putative heterozygous mutation in the COPA gene (c.841C>T; p.Arg281.Trp), thus confirming the diagnosis of COPA syndrome.

\section{CONCLUSION}

Here we report the first case of COPA syndrome in Brazil. Clinical signs of severe destructive arthritis and lung involvement should be considered as red flags for genetic screening in the context of juvenile chronic arthritis. All patients identified to date have been reported to harbor mutations in the coding region of the WD40 domain (amino acid residues 230-240), which is responsible for mRNA formation. Curiously, the mutation in the patient described herein is the first to occur outside the WD40 domain; accordingly, its impact on TH17 cell activation and type I interferon secretion must be further evaluated. COPA patients usually do not achieve clinical resolution using most common DMARDs drugs or through anti-TNF or anti-IL6 inhibition. However, Jak inhibitors do seem to present some efficacy in COPA syndrome, as reported in a case series involving the use of baricitinib and tofacitinib. 